\title{
SOFT SUPERSYMMETRY-BREAKING TERMS FROM SUPERGRAVITY AND SUPERSTRING MODELS ${ }^{a}$
}

\author{
A. BRIGNOLE \\ Theory Division, CERN, CH-1211 Geneva 23, Switzerland \\ L.E. IBÁÑNEZ \\ Departamento de Física Teórica, Universidad Autónoma de Madrid \\ Cantoblanco, 28049 Madrid, Spain \\ C. MUÑOZ ${ }^{b}$ \\ Department of Physics, Korea Advanced Institute of Science and Technology \\ Taejon 305-701, South Korea
}

\begin{abstract}
We review the origin of soft supersymmetry-breaking terms in $N=1$ supergravity models of particle physics. We first consider general formulae for those terms in general models with a hidden sector breaking supersymmetry at an intermediate energy scale. The results for some simple models are given. We then consider the results obtained in some simple superstring models in which particular assumptions about the origin of supersymmetry breaking are made. These are models in which the seed of supersymmetry breaking is assumed to be originated in the dilaton/moduli sector of the theory.
\end{abstract}

CERN-TH/97-143

FTUAM $97 / 7$

KAIST-TH-97/7

hep-ph/9707209

\footnotetext{
${ }^{a}$ To appear in the book 'Perspectives on Supersymmetry', World Scientific, Editor G. Kane. ${ }^{b}$ On leave of absence from Departamento de Física Teórica, Universidad Autónoma de Madrid, Cantoblanco, 28049 Madrid, Spain.
} 


\section{Introduction}

A phenomenological implementation of the idea of supersymmetry (SUSY) in the standard model requires the presence of SUSY breaking. There are essentially two large families of models in this context, depending on whether the scale of spontaneous SUSY breaking is high (of order $10^{10}-10^{13} \mathrm{GeV}$ ) or low (of order $1-10^{2} \mathrm{TeV}$ ). We will focus on the former possibility. The latter possibility, considered in other chapters of this book, has only recently received sufficient attention, since it was realized from the very first days of SUSY phenomenology that the existence of certain supertrace constraints in spontaneously broken SUSY theories made the building of realistic models quite complicated. Possible solutions to these early difficulties are discussed elsewhere and we are not going to discuss them further here.

A more pragmatic attitude to the issue of SUSY breaking is the addition of explicit soft SUSY-breaking terms of the appropriate size (of order $10^{2}$ $10^{3} \mathrm{GeV}$ ) in the Lagrangian and with appropriate flavour symmetries to avoid dangerous flavour-changing neutral currents (FCNC) transitions. The problem with this pragmatic attitude is that, taken by itself, lacks any theoretical explanation. Supergravity theories provide an attractive context that can justify such a procedure. Indeed, if one considers the SUSY standard model and couples it to $N=1$ supergravity, the spontaneous breaking of local SUSY in a hidden sector generates explicit soft SUSY-breaking terms of the required form in the effective low-energy Lagrangiand. If. If SUSY is broken at a scale $\Lambda_{S}$, the soft terms have a scale of order $\Lambda_{S}^{2} / M_{\text {Planck. }}$. Thus one obtains the required size if SUSY is broken at an intermediate scale $\Lambda_{S} \sim 10^{10} \mathrm{GeV}$, as mentioned above. Large classes of supergravity models, as we discuss in section 2 , give rise to universal soft SUSY-breaking terms, providing for an understanding of FCNC supression. In the last few years it has often been stated in the literature that this class of supergravity models have a flavour-changing problem. We think more appropriate to say that some particular models get interesting constraints from FCNC bounds. A generic statement like that seems unjustified, since it is usually based on a strong assumption, i.e. the existence of a region in between the grand unified theory (GUT) scale and the Planck (or superstring) scale in which important flavour non-diagonal renormalization effects take place.

Recently there have been studies of supergravity models obtained in particularly simple classes of superstring compactifications 3 . Such heterotic models have a natural hidden sector built-in: the complex dilaton field $S$ and the complex moduli fields $T_{i}$. These gauge singlet fields are generically present in four-dimensional models: the dilaton arises from the gravitational sector of the 
theory and the moduli parametrize the size and shape of the compactified variety. Assuming that the auxiliary fields of those multiplets are the seed of SUSY breaking, interesting predictions for this simple class of models are obtained. These are reviewed in section 3. The analysis does not assume any specific SUSY-breaking mechanism. We leave section 4 for some final comments and additional references to recent work.

\section{Soft terms from supergravity}

\subsection{General computation of soft terms}

The full $\mathrm{N}=1$ supergravity Lagrangian 1 (up to two derivatives) is specified in terms of two functions which depend on the chiral superfields $\phi_{M}$ of the theory (denoted by the same symbol as their scalar components): the analytic gauge kinetic function $f_{a}\left(\phi_{M}\right)$ and the real gauge-invariant Kähler function $G\left(\phi_{M}, \phi_{M}^{*}\right) . f_{a}$ determines the kinetic terms for the fields in the vector multiplets and in particular the gauge coupling constant, $R e f_{a}=1 / g_{a}^{2}$. The subindex $a$ is associated with the different gauge groups of the theory since in general $\mathcal{G}=\prod_{a} \mathcal{G}_{a}$. For example, in the case of the pure SUSY standard model coupled to supergravity, a would correspond to $S U(3)_{c}, S U(2)_{L}, U(1)_{Y} \cdot G$ is a combination of two functions

$$
G\left(\phi_{M}, \phi_{M}^{*}\right)=K\left(\phi_{M}, \phi_{M}^{*}\right)+\log \left|W\left(\phi_{M}\right)\right|^{2},
$$

where $K$ is the Kähler potential, $W$ is the complete analytic superpotential, and we use from now on the standard supergravity mass units where $M_{P} \equiv$ $M_{\text {Planck }} / \sqrt{8 \pi}=1$. $W$ is related with the Yukawa couplings (which eventually determine the fermion masses) and also includes possibly non-perturbative effects

$$
W=\hat{W}\left(h_{m}\right)+\frac{1}{2} \mu_{\alpha \beta}\left(h_{m}\right) C^{\alpha} C^{\beta}+\frac{1}{6} Y_{\alpha \beta \gamma}\left(h_{m}\right) C^{\alpha} C^{\beta} C^{\gamma}+\ldots,
$$

where we assume two different types of scalar fields $\phi_{M}=h_{m}, C^{\alpha}: C^{\alpha}$ correspond to the observable sector and in particular include the SUSY standard model fields, while $h_{m}$ correspond to a hidden sector. The latter fields may develop large $\left(\gg M_{W}\right)$ vacuum expectation values (VEVs) and are responsible for SUSY breaking if some auxiliary components $F^{m}$ (see below) develop nonvanishing VEVs. The ellipsis indicates terms of higher order in $C^{\alpha}$ whose coefficients are suppressed by negative powers of $M_{P}$. The second derivative of $K$ determines the kinetic terms for the fields in the chiral supermultiplets and is thus important for obtaining the proper normalization of the fields. 
Expanding in powers of $C^{\alpha}$ and $C^{* \bar{\alpha}}$ we have

$$
\begin{aligned}
K= & \hat{K}\left(h_{m}, h_{m}^{*}\right)+\tilde{K}_{\bar{\alpha} \beta}\left(h_{m}, h_{m}^{*}\right) C^{* \bar{\alpha}} C^{\beta} \\
& +\left[\frac{1}{2} Z_{\alpha \beta}\left(h_{m}, h_{m}^{*}\right) C^{\alpha} C^{\beta}+\text { h.c. }\right]+\ldots,
\end{aligned}
$$

where the ellipsis indicates terms of higher order in $C^{\alpha}$ and $C^{* \bar{\alpha}}$. Notice that the coefficients $\tilde{K}_{\bar{\alpha} \beta}, Y_{\alpha \beta \gamma}, \mu_{\alpha \beta}$, and $Z_{\alpha \beta}$ which appear in (2) and (3) may depend on the hidden sector fields in general. The bilinear terms associated with $\mu_{\alpha \beta}$ and $Z_{\alpha \beta}$ are often forbidden by gauge invariance in specific models, but they may be relevant in order to solve the so-called $\mu$ problem in the context of the minimal supersymmetric standard model (MSSM), as we will discuss below. In this case the two Higgs doublets, which are necessary to break the electroweak symmetry, have opposite hypercharges. Therefore those terms are allowed and may generate both the $\mu$ parameter and the corresponding soft bilinear term.

The (F part of the) tree-level supergravity scalar potential, which is crucial to analyze the breaking of SUSY, is given by

$$
V\left(\phi_{M}, \phi_{M}^{*}\right)=e^{G}\left(G_{M} K^{M \bar{N}} G_{\bar{N}}-3\right)=\left(\bar{F}^{\bar{N}} K_{\bar{N} M} F^{M}-3 e^{G}\right),
$$

where $G_{M} \equiv \partial_{M} G \equiv \partial G / \partial \phi_{M}$ and the matrix $K^{M \bar{N}}$ is the inverse of the Kähler metric $K_{\bar{N} M} \equiv \partial_{\bar{N}} \partial_{M} K$. We have also written $V$ as a function of the $\phi_{M}$ auxiliary fields, $F^{M}=e^{G / 2} K^{M \bar{P}} G_{\bar{P}}$. When, at the minimum of the scalar potential, some of the hidden sector fields $h_{m}$ acquire VEVs in such a way that at least one of their auxiliary fields $\left(\hat{K}^{m \bar{n}}\right.$ is the inverse of the hidden field metric $\hat{K}_{\bar{n} m}$ )

$$
F^{m}=e^{G / 2} \hat{K}^{m \bar{n}} G_{\bar{n}}
$$

is non-vanishing, then SUSY is spontaneously broken and soft SUSY-breaking terms are generated in the observable sector. Let us remark that, for simplicity, we are assuming vanishing D-term contributions to SUSY breaking. When this is not the case, their effects on soft terms can be found e.g. in $\theta_{\text {. The }}$ goldstino, which is a combination of the fermionic partners of the above fields, is swallowed by the gravitino via the superHiggs effect. The gravitino becomes massive and its mass

$$
m_{3 / 2}=e^{G / 2}
$$

sets the overall scale of the soft parameters. 


\section{General results}

Using the above information, the soft SUSY-breaking terms in the observable sector can be computed. They are obtained by replacing $h_{m}$ and their auxiliary fields $F^{m}$ by their VEVs in the supergravity Lagrangian and taking the so-called flat limit where $M_{P} \rightarrow \infty$ but $m_{3 / 2}$ is kept fixed. Then the nonrenormalizable gravity corrections are formally eliminated and one is left with a global SUSY Lagrangian plus a set of soft SUSY-breaking terms. On the one hand, from the fermionic part of the supergravity Lagrangian, soft gaugino masses for the canonically normalized gaugino fields can be obtained

$$
M_{a}=\frac{1}{2}\left(R e f_{a}\right)^{-1} F^{m} \partial_{m} f_{a}
$$

as well as the un-normalized Yukawa couplings of the observable sector fermions and the SUSY un-normalized masses of some of them (those with bilinear terms either in the superpotential or in the Kähler potential, e.g. the Higgsinos in the case of the MSSM)

$$
\begin{aligned}
Y_{\alpha \beta \gamma}^{\prime} & =\frac{\hat{W}^{*}}{|\hat{W}|} e^{\hat{K} / 2} Y_{\alpha \beta \gamma}, \\
\mu_{\alpha \beta}^{\prime} & =\frac{\hat{W}^{*}}{|\hat{W}|} e^{\hat{K} / 2} \mu_{\alpha \beta}+m_{3 / 2} Z_{\alpha \beta}-\bar{F}^{\bar{m}} \partial_{\bar{m}} Z_{\alpha \beta} .
\end{aligned}
$$

On the other hand, scalar soft terms arise from the expansion of the supergravity scalar potential (4)

$$
V_{\text {soft }}=m_{\bar{\alpha} \beta}^{\prime 2} C^{* \bar{\alpha}} C^{\beta}+\left(\frac{1}{6} A_{\alpha \beta \gamma}^{\prime} C^{\alpha} C^{\beta} C^{\gamma}+\frac{1}{2} B_{\alpha \beta}^{\prime} C^{\alpha} C^{\beta}+\text { h.c. }\right) .
$$

In the most general case, when hidden and observable sector matter metrics are not diagonal, the un-normalized soft scalar masses, trilinear and bilinear parameters are given respectively by

$$
\begin{aligned}
m_{\bar{\alpha} \beta}^{\prime 2}= & \left(m_{3 / 2}^{2}+V_{0}\right) \tilde{K}_{\bar{\alpha} \beta} \\
& -\bar{F}^{\bar{m}}\left(\partial_{\bar{m}} \partial_{n} \tilde{K}_{\bar{\alpha} \beta}-\partial_{\bar{m}} \tilde{K}_{\bar{\alpha} \gamma} \tilde{K}^{\gamma \bar{\delta}} \partial_{n} \tilde{K}_{\bar{\delta} \beta}\right) F^{n}, \\
A_{\alpha \beta \gamma}^{\prime}= & \frac{\hat{W}^{*}}{|\hat{W}|} e^{\hat{K} / 2} F^{m}\left[\hat{K}_{m} Y_{\alpha \beta \gamma}+\partial_{m} Y_{\alpha \beta \gamma}\right. \\
& \left.-\left(\tilde{K}^{\delta \bar{\rho}} \partial_{m} \tilde{K}_{\bar{\rho} \alpha} Y_{\delta \beta \gamma}+(\alpha \leftrightarrow \beta)+(\alpha \leftrightarrow \gamma)\right)\right],
\end{aligned}
$$




$$
\begin{aligned}
B_{\alpha \beta}^{\prime}= & \frac{\hat{W}^{*}}{|\hat{W}|} e^{\hat{K} / 2}\left\{F ^ { m } \left[\hat{K}_{m} \mu_{\alpha \beta}+\partial_{m} \mu_{\alpha \beta}\right.\right. \\
& \left.\left.-\left(\tilde{K}^{\delta \bar{\rho}} \partial_{m} \tilde{K}_{\bar{\rho} \alpha} \mu_{\delta \beta}+(\alpha \leftrightarrow \beta)\right)\right]-m_{3 / 2} \mu_{\alpha \beta}\right\} \\
& +\left(2 m_{3 / 2}^{2}+V_{0}\right) Z_{\alpha \beta}-m_{3 / 2} \bar{F}^{m} \partial_{\bar{m}} Z_{\alpha \beta} \\
& +m_{3 / 2} F^{m}\left[\partial_{m} Z_{\alpha \beta}-\left(\tilde{K}^{\delta \bar{\rho}} \partial_{m} \tilde{K}_{\bar{\rho} \alpha} Z_{\delta \beta}+(\alpha \leftrightarrow \beta)\right)\right] \\
& -\bar{F}^{\bar{m}} F^{n}\left[\partial_{\bar{m}} \partial_{n} Z_{\alpha \beta}-\left(\tilde{K}^{\delta \bar{\rho}} \partial_{n} \tilde{K}_{\bar{\rho} \alpha} \partial_{\bar{m}} Z_{\delta \beta}+(\alpha \leftrightarrow \beta)\right)\right],
\end{aligned}
$$

where $\tilde{K}^{\alpha \bar{\beta}}$ is the inverse of the observable matter metric $\tilde{K}_{\bar{\beta} \gamma} \cdot V_{0}$ is the VEV of the scalar potential (1), i.e. the tree-level cosmological constant

$$
V_{0}=\bar{F}^{\bar{m}} \hat{K}_{\bar{m} n} F^{n}-3 m_{3 / 2}^{2} .
$$

It has a bearing on measurable quantities like scalar masses and therefore it is preferable to leave it undetermined (see 6.6 . Notice that, after normalizing the fields to get canonical kinetic terms, the first piece in (11) will lead to universal diagonal soft masses but the second piece will generically induce off-diagonal contributions. Actually, universality is a desirable property not only to reduce the number of independent parameters in SUSY models, but also for phenomenological reasons, particularly to avoid FCNC. The latter is a low-energy phenomenological constraint that must be satisfied by any supergravity model. It is worth mentioning in this context that one-loop corrections to the soft parameters (7), (11), (12) and (13) have recently been computed in 1 . They may induce FCNC phenomena even when the tree-level computation gives a universal soft mass. Also a discussion about the loop effects on the contribution of the cosmological constant to the soft terms can be found there. Concerning the $A$ and $B$ parameters, notice that we have not factored out the Yukawa couplings and mass terms respectively as usual, since proportionality is not guaranteed in (12) and (13). Finally, from (5) and (5), one can easily see that the (tree-level) soft parameters in (7), (11) and (12) are generically $\mathcal{O}\left(m_{3 / 2}\right)$, as mentioned above. A departure from this result is only possible when some of them vanish. We will mention below some examples of this type, i.e. the case of no-scale supergravity models and special limits of superstring models.

The $\mu$ problem

The set of mass parameters in the MSSM Higgs potential includes, besides $\mathcal{O}\left(m_{3 / 2}^{2}\right)$ soft masses, the square of $\mu_{\alpha \beta}^{\prime}$ (9) and the $B$ parameter (13), which is $\mathcal{O}\left(m_{3 / 2} \mu_{\alpha \beta}^{\prime}\right)$. In order to have correct electroweak symmetry breaking, the 
SUSY mass term $\mu_{\alpha \beta}^{\prime}$ should also be $\mathcal{O}\left(m_{3 / 2}\right)$. This is the so-called $\mu$ problemb. In this respect, notice that $\mu_{\alpha \beta}^{\prime}=\mathcal{O}\left(m_{3 / 2}\right)$ is naturally achieved in the presence of a non-vanishing $Z_{\alpha \beta}$ in the Kähler potential 1 , 11 . The other possible source of the mass $\mu_{\alpha \beta}^{\prime}$ is the SUSY mass $\mu_{\alpha \beta}$ in the superpotential 2 . This case is more involved since in principle the natural scale of $\mu_{\alpha \beta}$ would be $M_{P}$. However, a possible solution can he obtained if the superpotential contains e.g. a non-renormalizable term 11 , 23

$$
\lambda\left(h_{m}\right) \hat{W}\left(h_{m}\right) H_{1} H_{2},
$$

characterized by the coupling $\lambda$, which mixes the observable sector with the hidden sector. Since $m_{3 / 2}=e^{G / 2}=e^{\hat{K} / 2}|\hat{W}|$, if that term exists then an effective $\mu$ parameter $\mathcal{O}\left(m_{3 / 2}\right)$ is generated dynamically when $h_{m}$ acquire VEVs:

$$
\mu=\lambda\left(h_{m}\right) \hat{W}\left(h_{m}\right) .
$$

We should add that both mechanisms to generate $\mu_{\alpha \beta}^{\prime}$, a bilinear term in the Kähler potential or in the superpotential, could be present simultaneously. Notice also that the two mechanisms are equivalent if $Z$ depends only on $h_{m}$ (not on $h_{m}^{*}$ ). Indeed, in that case the supergravity theory is equivalent to the one with a Kähler potential $K$ without the terms $Z H_{1} H_{2}+$ h.c. and a superpotential $W e^{Z H_{1} H_{2}}$, since the $G$ function (11) is the same for both. After expanding the exponential, the superpotential will have a contribution $Z \hat{W} H_{1} H_{2}$, i.e. a term of the type (15). Finally, let us mention that several new sources of the $\mu$ term due to loop effects on (9) which are naturally of order the weak scale, have recently been computed in 2 .

We recall that the solutions mentioned here in order to solve the $\mu$ problem are naturally present in superstring models. For instance, in large classes of superstring models the Kähler potential does contain bilinear terms analytic in the observable fields as in (3), with specific coefficients $Z_{\alpha \beta}{ }_{13} 44.15$, so that a $\mu$ parameter may be naturally generated. Concerning superpotential contributions, we recall that a 'direct' $\mu H_{1} H_{2}$ term in $W$ (2) is naturally absent (otherwise the natural scale for $\mu$ would be $M_{P}$ ), since in supergravity models deriving from superstring theory mass terms for light fields are forbidden in the superpotential by scale invariance of the theory. However, the superpotential (2) pay well contain an 'effective' $\mu H_{1} H_{2}$ term, e.g. a term of the type (15) 11,15 induced by non-perturbative SUSY-breaking mechanisms like gaugino-squark condensation in the hidden sector.

The low-energy spectrum

The results (7), (8), (9), (11), (12) and (13) should be understood as being valid at some high scale $\mathcal{O}\left(M_{P}\right)$ and the standard RGEs must be used to obtain 
the low-energy values. Although the SUSY spectrum will depend in general on the details of $S U(2)_{L} \times U(1)_{Y}$ breaking, there are several particles whose mass is rather independent of those details and is mostly given by the boundary conditions and the renormalization group running. In particular, neglecting all Yukawa couplings except the one of the top, that is the case of the gluino $g$, all the squarks (except stops and left sbottom) $Q_{L}=\left(u_{L}, d_{L}\right), u_{L}^{c}, d_{L}^{c}$ and all the sleptons $L_{L}=\left(v_{L}, e_{L}\right), e_{L}^{c}$. For all these particles one can write explicit expressions for the masses in terms of the soft parameters (after normalizing the fields to get canonical kinetic terms). For instance, assuming that gauginos have a common initial mass (e.g. due to a universal $f$ function) and that there is nothing but the MSSM in between the weak scale and the Planck scale, one obtains the approximate numerical expressions:

$$
\begin{aligned}
M_{g}^{2}\left(M_{Z}\right) & \simeq 9.8 M^{2} \\
m_{Q_{L}}^{2}\left(M_{Z}\right) & \simeq m_{Q_{L}}^{2}+8.3 M^{2} \\
m_{u_{L}^{c}, d_{L}^{c}}^{2}\left(M_{Z}\right) & \simeq m_{u_{L}^{c}, d_{L}^{c}}^{2}+8 M^{2} \\
m_{L_{L}}^{2}\left(M_{Z}\right) & \simeq m_{L_{L}}^{2}+0.7 M^{2} \\
m_{e_{L}^{c}}^{2}\left(M_{Z}\right) & \simeq m_{e_{L}^{c}}^{2}+0.23 M^{2}
\end{aligned}
$$

where the second term in the expression of the scalar masses is the effect of gaugino loop contributions. In the above formulae we have neglected the scalar potential D-term contributions, which are normally small compared to the terms above, and the contribution of the $U(1)_{Y}$ D-term in the RGEs of scalar masses. These may be found e.g. in 16 .

\subsection{Supergravity models}

We now specialize the above general discussion to the case of supergravity models where the observable (here MSSM) matter fields have diagonal metric:

$$
\tilde{K}_{\bar{\alpha} \beta}\left(h_{m}, h_{m}^{*}\right)=\delta_{\bar{\alpha} \beta} \tilde{K}_{\alpha}\left(h_{m}, h_{m}^{*}\right) .
$$

This possibility is particularly interesting due to its simplicity and also for phenomenological reasons related to the absence of FCNC in the effective lowenergy theory (see 6.17 18.19.20.6 for a discussion on this point). Besides, the supergravity models that will be studied below correspond to this situation. Then the Kähler potential (3), to lowest order in the observable fields $C^{\alpha}$, and the superpotential (2) have the form

$$
K=\hat{K}\left(h_{m}, h_{m}^{*}\right)+\tilde{K}_{\alpha}\left(h_{m}, h_{m}^{*}\right) C^{* \bar{\alpha}} C^{\alpha}+\left[Z\left(h_{m}, h_{m}^{*}\right) H_{1} H_{2}+h . c .\right]
$$




$$
\begin{aligned}
W= & \hat{W}\left(h_{m}\right)+\mu\left(h_{m}\right) H_{1} H_{2}+\sum_{\text {generations }}\left[Y_{u}\left(h_{m}\right) Q_{L} H_{2} u_{L}^{c}\right. \\
& \left.+Y_{d}\left(h_{m}\right) Q_{L} H_{1} d_{L}^{c}+Y_{e}\left(h_{m}\right) L_{L} H_{1} e_{L}^{c}\right],
\end{aligned}
$$

where $C^{\alpha}=Q_{L}, u_{L}^{c}, d_{L}^{c}, L_{L}, e_{L}^{c}, H_{1}, H_{2}$, and we have taken for simplicity diagonal Yukawa couplings $\left(Y_{\alpha \beta \gamma}=Y_{u}, Y_{d}, Y_{e}\right.$, in a self-explanatory notation). Now the form of the effective soft Lagrangian obtained from (7) and (10) is given by

$$
\begin{aligned}
\mathcal{L}_{\text {soft }}= & \frac{1}{2}\left(M_{a} \widehat{\lambda}^{a} \widehat{\lambda}^{a}+\text { h.c. }\right)-m_{\alpha}^{2} \widehat{C}^{* \bar{\alpha}} \widehat{C}^{\alpha} \\
& -\left(\frac{1}{6} A_{\alpha \beta \gamma} \widehat{Y}_{\alpha \beta \gamma} \widehat{C}^{\alpha} \widehat{C}^{\beta} \widehat{C}^{\gamma}+B \widehat{\mu} \widehat{H}_{1} \widehat{H}_{2}+\text { h.c. }\right),
\end{aligned}
$$

with

$$
\begin{aligned}
m_{\alpha}^{2}= & \left(m_{3 / 2}^{2}+V_{0}\right)-\bar{F}^{\bar{m}} F^{n} \partial_{\bar{m}} \partial_{n} \log \tilde{K}_{\alpha} \\
A_{\alpha \beta \gamma}= & F^{m}\left[\hat{K}_{m}+\partial_{m} \log Y_{\alpha \beta \gamma}-\partial_{m} \log \left(\tilde{K}_{\alpha} \tilde{K}_{\beta} \tilde{K}_{\gamma}\right)\right] \\
B= & \widehat{\mu}^{-1}\left(\tilde{K}_{H_{1}} \tilde{K}_{H_{2}}\right)^{-1 / 2}\left\{\frac { \hat { W } ^ { * } } { | \hat { W } | } e ^ { \hat { K } / 2 } \mu \left(F ^ { m } \left[\hat{K}_{m}+\partial_{m} \log \mu\right.\right.\right. \\
& \left.\left.-\partial_{m} \log \left(\tilde{K}_{H_{1}} \tilde{K}_{H_{2}}\right)\right]-m_{3 / 2}\right) \\
& +\left(2 m_{3 / 2}^{2}+V_{0}\right) Z-m_{3 / 2} \bar{F}^{\bar{m}} \partial_{\bar{m}} Z \\
& +m_{3 / 2} F^{m}\left[\partial_{m} Z-Z \partial_{m} \log \left(\tilde{K}_{H_{1}} \tilde{K}_{H_{2}}\right)\right] \\
& \left.-\bar{F}^{\bar{m}} F^{n}\left[\partial_{\bar{m}} \partial_{n} Z-\partial_{\bar{m}} Z \partial_{n} \log \left(\tilde{K}_{H_{1}} \tilde{K}_{H_{2}}\right)\right]\right\}
\end{aligned}
$$

where $\widehat{C}^{\alpha}$ and $\widehat{\lambda}^{a}$ are the scalar and gaugino canonically normalized fields respectively

$$
\begin{aligned}
\widehat{C}^{\alpha} & =\tilde{K}_{\alpha}^{1 / 2} C^{\alpha}, \\
\widehat{\lambda}^{a} & =\left(\operatorname{Re} f_{a}\right)^{1 / 2} \lambda^{a},
\end{aligned}
$$

and the rescaled Yukawa couplings and $\mu$ parameter

$$
\begin{aligned}
\widehat{Y}_{\alpha \beta \gamma} & =Y_{\alpha \beta \gamma} \frac{\hat{W}^{*}}{|\hat{W}|} e^{\hat{K} / 2}\left(\tilde{K}_{\alpha} \tilde{K}_{\beta} \tilde{K}_{\gamma}\right)^{-1 / 2} \\
\widehat{\mu} & =\left(\frac{\hat{W}^{*}}{|\hat{W}|} e^{\hat{K} / 2} \mu+m_{3 / 2} Z-\bar{F}^{\bar{m}} \partial_{\bar{m}} Z\right)\left(\tilde{K}_{H_{1}} \tilde{K}_{H_{2}}\right)^{-1 / 2},
\end{aligned}
$$


have been factored out in the $A$ and $B$ terms as usual.

Now we are ready to study specific supergravity models. As follows from the above discussion, the particular values of the soft parameters depend on the type of supergravity theory from which the MSSM derives and, in general, on the mechanism of SUSY breaking (through the presence of $\hat{W}\left(h_{m}\right)$ in $m_{3 / 2}$ and $\mathrm{F}$ terms). However, it is still possible to learn things about soft parameters without knowing all the details of SUSY breaking. In order to show this, let us consider two simple and interesting supergravity models studied extensively in the literature: minimal supergravity and no-scale supergravity.

i) Minimal supergravity

This model corresponds to use the form of $K$ that leads to minimal (canonical) kinetic terms in the supergravity Lagrangian, namely

$$
\tilde{K}_{\alpha}\left(h_{m}, h_{m}^{*}\right)=1
$$

in (19). Then, irrespective of the SUSY-breaking mechanism, the scalar masses and the $A, B$ parameters can be straightforwardly computed using (22), 23) and (24)

$$
\begin{aligned}
m_{\alpha}^{2}= & m_{3 / 2}^{2}+V_{0} \\
A_{\alpha \beta \gamma}= & F^{m}\left(\hat{K}_{m}+\partial_{m} \log Y_{\alpha \beta \gamma}\right) \\
B= & \widehat{\mu}^{-1}\left\{\frac{\hat{W}^{*}}{|\hat{W}|} e^{\hat{K} / 2} \mu\left[F^{m}\left(\hat{K}_{m}+\partial_{m} \log \mu\right)-m_{3 / 2}\right]\right. \\
& +\left(2 m_{3 / 2}^{2}+V_{0}\right) Z+m_{3 / 2}\left(F^{m} \partial_{m} Z-\bar{F}^{\bar{m}} \partial_{\bar{m}} Z\right) \\
& \left.-\bar{F}^{\bar{m}} F^{n} \partial_{\bar{m}} \partial_{n} Z\right\},
\end{aligned}
$$

where

$$
\widehat{\mu}=\frac{\hat{W}^{*}}{|\hat{W}|} e^{\hat{K} / 2} \mu+m_{3 / 2} Z-\bar{F}^{\bar{m}} \partial_{\bar{m}} Z .
$$

Notice that the scalar masses are automatically universal in this model. Further simplifications can be obtained if $Z=0$ and if the superpotential parameters $Y_{\alpha \beta \gamma}$ and $\mu$ do not depend on the hidden sector fields. Under such assumptions, which are common in the literature, (31) and (32) generate universal $A$ parameters, as well as the relation

$$
B=A-m_{3 / 2} .
$$


Furthermore, if we assume $V_{0}=0$, then $m \equiv m_{\alpha}=m_{3 / 2}$ and the well known result for the $B$ parameter, $B=A-m$, is recovered. This supergravity model is attractive for its simplicity and for the natural explanation that it offers to the universality of the soft scalar masses.

We remark that although minimal (canonical) kinetic terms for hidden matter, $\hat{K}\left(h_{m}, h_{m}^{*}\right)=\sum_{m} h_{m} h_{m}^{*}$, are also usually assumed, we have seen that it is not a necessary condition in order to obtain the above results. Concerning the kinetic terms for vector multiplets, it can be seen from (7) that the minimal (canonical) choice $f_{a}=$ const . is not phenomenologically interesting, since it implies $M_{a}=0$. Nonvanishing and universal gaugino masses can be obtained if all the $f_{a}$ have the same dependence on the hidden sector fields, i.e. $f_{a}\left(h_{m}\right)=$ $c_{a} f\left(h_{m}\right)$ for the different gauge group factors of the theory. This is in fact what happens, at tree level, in supergravity models deriving from superstring theory, as we will see in the next section. As an additional comment, we stress that relation (34) depends on the particular mechanism that is used to generate the $\mu$ parameter. As a counter-example, notice that if one takes e.g. an $h_{m}$-dependent $\mu$ as in (16) with $\lambda=$ const., instead of taking $\mu=$ const., then (32) gives

$$
B=2 m_{3 / 2}+\frac{V_{0}}{m_{3 / 2}}
$$

with $\widehat{\mu}=m_{3 / 2} \lambda$ from (33). Thus the relation (34) does not hold. The above result (35) can be obtained also if one takes $\mu=0$ in the superpotential (20) and $Z=$ const. in the Kähler potential (19). This also follows from our discussion above about the equivalence between the two mechanisms when $Z$ is an analytic function.

ii) No-scale supergravity

In no-scale supergravity models 21, after the spontaneous breaking of SUSY, the tree-level potential vanishes identically along some directions. A simple example of this type of models has just one hidden field $h$, a Kähler potential (19) with

$$
\hat{K}=-3 \log \left(h+h^{*}\right), \tilde{K}_{\alpha}=\left(h+h^{*}\right)^{-1},
$$

and a superpotential (20) with a hidden field independent $\hat{W}$, i.e. $\hat{W}=$ const. Then, the attractive result of a vanishing (flat) tree-level effective potential for the hidden sector (14) is obtained

$$
V_{0}=0,
$$

for all VEVs of $h$. On the other hand, the soft parameters, using (22), (23) 
and (24), are given by

$$
\begin{aligned}
m_{\alpha}^{2}= & 0, \\
A_{\alpha \beta \gamma}= & -m_{3 / 2}\left(h+h^{*}\right) \partial_{h} \log Y_{\alpha \beta \gamma}, \\
B= & -\hat{\mu}^{-1} m_{3 / 2}\left(h+h^{*}\right)^{2}\left\{\frac{\hat{W}^{*}}{|\hat{W}|}\left(h+h^{*}\right)^{-3 / 2} \partial_{h} \mu\right. \\
& \left.+m_{3 / 2}\left[\partial_{h^{*}} Z+\partial_{h} Z+\left(h+h^{*}\right) \partial_{h} \partial_{h^{*}} Z\right]\right\},
\end{aligned}
$$

where

$$
\widehat{\mu}=\left(h+h^{*}\right)\left[\frac{\hat{W}^{*}}{|\hat{W}|}\left(h+h^{*}\right)^{-3 / 2} \mu+m_{3 / 2} Z+m_{3 / 2}\left(h+h^{*}\right) \partial_{h^{*}} Z\right] .
$$

Assuming now that the $\mu$ and $Z$ coefficients and the Yukawa couplings are hidden field independent, the well known result for the soft parameters is recovered:

$$
m_{\alpha}=A_{\alpha \beta \gamma}=B=0 .
$$

Although the above parameters are vanishing at the high scale, gaugino masses (7) can induce non-vanishing values at the electroweak scale due to radiative corrections.

In conclusion, both supergravity models considered in this section are interesting and give rise to concrete predictions for the soft parameters. However, one can think of many possible supergravity models (with different $K, W$ and $f$ ) leading to different results for the soft terms. This arbitrariness, as we will see in the next section, can be ameliorated in supergravity models deriving from superstring theory, where $K, f$, and the hidden sector are more constrained. We can already anticipate, however, that in such a context the kinetic terms are generically not canonical. Besides, although Kähler potentials of the no-scale type may appear at tree-level, the superpotentials are in general hidden field dependent. Moreover, the Yukawa couplings $Y_{\alpha \beta \gamma}$ and the bilinear coefficients $\mu$ and $Z$ are also generically hidden field dependent.

Finally, we remark that further constraints on the soft parameter space of the MSSM can be obtained if one wishes to avoid low-energy charge and color breaking minima deeper than the standard vacuum22. On these grounds, and assuming also radiative symmetry breaking with nothing but the MSSM in between the weak scale and the Planck scale, e.g. large regions in the parameter space $(m, M, A, B)$ of the minimal supergravity model i) are forbidden. In the limiting case $m=0$ the whole parameter space turns out to be excluded. This 
has obvious implications, e.g. for the no-scale supergravity model ii). If the same kind of analysis is applied to the soft parameters of superstring models, again strong constraints can be obtained, as we will comment below.

\section{Soft terms from superstring theory}

\subsection{General parametrization of SUSY breaking}

We are going to consider $\mathrm{N}=1$ four-dimensional superstrings where the rôle of hidden sector fields is effectively played by $r$ moduli fields $T_{i}, i=1, \ldots, r$ and the dilaton field $S$, i.e. $h_{m}=S, T_{i}$ following the notation of the previous section. We recall that we are denoting the $T$ - and $U$-type (Kähler class and complex structure in the Calabi-Yau language) moduli collectively by $T_{i}$. The associated effective $\mathrm{N}=1$ supergravity Kähler potentials (3), to lowest order in the matter fields, are of the type:

$$
\begin{aligned}
K= & \hat{K}\left(S, S^{*}, T_{i}, T_{i}^{*}\right)+\tilde{K}_{\bar{\alpha} \beta}\left(T_{i}, T_{i}^{*}\right) C^{* \bar{\alpha}} C^{\beta} \\
& +\left[\frac{1}{2} Z_{\alpha \beta}\left(T_{i}, T_{i}^{*}\right) C^{\alpha} C^{\beta}+\text { h.c. }\right]
\end{aligned}
$$

where at the superstring tree level

$$
\hat{K}\left(S, S^{*}, T_{i}, T_{i}^{*}\right)=-\log \left(S+S^{*}\right)+\hat{K}\left(T_{i}, T_{i}^{*}\right) .
$$

The first piece in (44) is the usual term corresponding to the complex dilaton $S$ that is present for any compactification. The second piece is the Kähler potential of the moduli fields, which in general depends on the compactification scheme and can be a complicated function. For the moment we leave it generic, but in the next subsection we will analyze some specific classes of superstring models where it has been computed. The same comment applies to $\tilde{K}_{\bar{\alpha} \beta}\left(T_{i}, T_{i}^{*}\right)$ and $Z_{\alpha \beta}\left(T_{i}, T_{i}^{*}\right)$. In the case of the superpotential (2),$Y_{\alpha \beta \gamma}\left(T_{i}\right)$ is also independent of $S$, but the non-perturbative contributions $\hat{W}\left(S, T_{i}\right)$ and $\mu_{\alpha \beta}\left(S, T_{i}\right)$ may depend in general on both $S$ and $T_{i}$. Finally, for any fourdimensional superstring the tree-level gauge kinetic function is independent of the moduli sector and is simply given by

$$
f_{a}=k_{a} S,
$$

where $k_{a}$ is the Kac-Moody level of the gauge factor. Usually (level one case) one takes $k_{3}=k_{2}=\frac{3}{5} k_{1}=1$, but this is irrelevant for our tree-level computation since $k_{a}$ will not contribute to the soft parameters.

As we will show below, it is important to know what fields, either $S$ or $T_{i}$, play the predominant role in the process of SUSY breaking. This will 
have relevant consequences in determining the pattern of soft parameters, and therefore the spectrum of physical particles 6 . That is why it is very useful to introduce the following parametrization, consistent with (14), for the VEVs of dilaton and moduli auxiliary fields

$$
\begin{aligned}
F^{S} & =\sqrt{3} C m_{3 / 2} K_{\bar{S} S}^{-1 / 2} \sin \theta e^{-i \gamma_{S}}, \\
F^{i} & =\sqrt{3} C m_{3 / 2} \cos \theta P^{i \bar{j}} \Theta_{\bar{j}},
\end{aligned}
$$

where the constant $C$ is defined as follows

$$
C^{2}=1+\frac{V_{0}}{3 m_{3 / 2}^{2}} .
$$

This parametrization is valid for the general case of off-diagonal moduli metric, since $P$ is a matrix canonically normalizing the moduli fields, i.e. $P^{\dagger} \hat{K} P=1$ where $\hat{K} \equiv \hat{K}_{\bar{i} j}$ and 1 stands for the unit matrix. The angle $\theta$ and the complex parameters $\Theta_{j}^{-}$just parametrize the direction of the goldstino in the $S, T_{i}$ field space (see below (5)) and $\sum_{j} \Theta_{j}^{*} \Theta_{\bar{j}}=1$. We have also allowed for the possibility of some of the theory (see 6,23,17,18,20.24 for a discussion on this point). Notice that if the tree-level cosmological constant $V_{0}$ is assumed to vanish, one has $C=1$, but we prefer for the moment to leave it undetermined as we did in the previous section (see below (14)).

Notice that such a phenomenological approach allows us to 'reabsorb' (or circumvent) our ignorance about the (nonperturbative) $S$ - and $T_{i}$ - dependent part of the superpotential (2), $\hat{W}\left(S, T_{i}\right)$, which is responsible for SUSY breaking.

It is now a straightforward exercise, plugging (43), (44), (45) and (46) into (7), (11), (12) and (13), to compute the soft SUSY-breaking parameters as functions of $\theta$ and $\Theta_{\bar{j}}$. On the one hand, since the tree-level gauge kinetic function is given for any four-dimensional superstring by (45), the tree-level gaugino masses are universal, independent of the moduli sector, and simply given by:

$$
M_{a}=\sqrt{3} C m_{3 / 2} \sin \theta e^{-i \gamma_{S}} .
$$

On the other hand, the bosonic soft parameters depend in general on the moduli sector (i.e. on the functions $\tilde{K}_{\bar{\alpha} \beta}, Z_{\alpha \beta}\left(T_{i}, T_{i}^{*}\right), \ldots$ and on the parameters $\cos \theta$ and $\Theta_{\bar{j}}$ ) and therefore they should be studied in the context of specific classes of superstring models. However, we will first focus on the very interesting limit $\cos \theta=0$, which corresponds to the case where the dilaton sector is the 
source of all the SUSY breaking (see (46) ) and the results are compactification independent.

\section{Dilaton SUSY breaking}

Since the dilaton couples in a universal manner to all particles, this limit is quite model independent 13 . Indeed, the expressions for all the soft parameters (except $B$ ) are quite simple and independent of the four-dimensional superstring considered. After canonically normalizing the fields, one obtains:

$$
\begin{aligned}
m_{\alpha}^{2}= & m_{3 / 2}^{2}+V_{0} \\
M_{a}= & \sqrt{3} C m_{3 / 2} e^{-i \gamma_{S}} \\
A_{\alpha \beta \gamma}= & -M_{a} \\
B= & \widehat{\mu}^{-1}\left(\tilde{K}_{H_{1}} \tilde{K}_{H_{2}}\right)^{-1 / 2}\left\{\frac{\hat{W}^{*}}{|\hat{W}|} e^{\hat{K} / 2} \mu m_{3 / 2}(-1\right. \\
& \left.\left.-\sqrt{3} C e^{-i \gamma_{S}}\left[1-\left(S+S^{*}\right) \partial_{S} \log \mu\right]\right)+Z\left(2 m_{3 / 2}^{2}+V_{0}\right)\right\}
\end{aligned}
$$

where

$$
\widehat{\mu}=\left(\frac{\hat{W}^{*}}{|\hat{W}|} e^{\hat{K} / 2} \mu+m_{3 / 2} Z\right)\left(\tilde{K}_{H_{1}} \tilde{K}_{H_{2}}\right)^{-1 / 2} .
$$

Although the general expression for $B$ is more involved than the ones of the other soft parameters, a considerable simplification occurs if $Z$ is the only source of the $\mu$ term. In this case $B$ reduces to

$$
B=2 m_{3 / 2}+\frac{V_{0}}{m_{3 / 2}} .
$$

and thus becomes independent of the four-dimensional superstring considered, as the other parameters. It is easy to check that the same result (54) is also obtained if $Z=0$ and the superpotential contains a $\mu$ coefficient of the form (16), where now $\mu=\lambda\left(T_{i}\right) \hat{W}\left(S, T_{i}\right)$. Notice that the expressions for $m_{\alpha}$ (49) and $B$ (54) coincide with the corresponding ones obtained in the minimal supergravity model i), (30) and (35) respectively. Furthermore, if $Z=0$ and $\partial_{S} \mu=0$, the expression for $B$ obtained from (52) coincides with the corresponding one (34) of the minimal supergravity model.

This dilaton-dominated scenario is attractive for its simplicity and for the natural explanation that it offers to the universality of the soft terms. For possible_explicit SUSY-breaking mechanisms where this limit might be obtained see 25. Because of the simplicity of this scenario, the low-energy predictions 
are quite precise 2662028. Assuming a vanishing cosmological constant and imposing, e.g. from the limits on the electric dipole moment of the neutron, $\gamma_{S}=0 \bmod \pi(49),(50)$ and (51) give 9

$$
m_{\alpha}=m_{3 / 2}, M_{a}= \pm \sqrt{3} m_{3 / 2}, A_{\alpha \beta \gamma}=-M_{a} .
$$

Since scalars are lighter than gauginos at the high scale and all mass ratios are fixed, at low-energy $\left(\sim M_{Z}\right)$ one finds the following mass ratios for the gluino, slepton and squark (except stops and left sbottom) masses

$$
M_{g}: m_{Q_{L}}: m_{u_{L}^{c}}: m_{d_{L}^{c}}: m_{L_{L}}: m_{e_{L}^{c}} \simeq 1: 0.94: 0.92: 0.92: 0.32: 0.24
$$

as can be computed e.g. from (17). Although squarks and sleptons have the same soft mass at the high scale, at low-energy the former are much heavier than the latter because of the gluino contribution to the renormalization of their masses. The rest of the spectrum is very dependent on the details of $S U(2)_{L} \times U(1)_{Y}$ breaking and therefore on the values of $B$ and $\widehat{\mu}$. For $B=$ $2 m_{\mathrm{3} / 2}$ (see(54)) and $\mu$ treated as a free parameter this analysis can be found in 26. Modifications to this scenario due to the effect of possible superstring non-perturbative corrections to the Kähler potential can be found in 30 .

It is worth noticing here that, although the value of $\widehat{\mu}(53)$ is compactification dependent even in this dilaton-dominated scenario, the simple result $\widehat{\mu}=m_{3 / 2}$ can be obtained in any compactification scheme where the source of $\widehat{\mu}$ is a $Z$ term in the Kähler potential fulfilling the property $Z=\left(\tilde{K}_{H_{1}} \tilde{K}_{H_{2}}\right)^{1 / 2}$. In fact, we will see in the next subsection that this is the case of some classes of orbifold models. Notice that, when such a property holds, the whole SUSY spectrum depends only on one parameter, $m_{3 / 2}$, since

$$
m_{\alpha}=m_{3 / 2}, M_{a}= \pm \sqrt{3} m_{3 / 2}, A_{\alpha \beta \gamma}=-M_{a}, B=2 m_{3 / 2}, \widehat{\mu}=m_{3 / 2} .
$$

Besides, this parameter can be fixed from the phenomenological requirement of correct electroweak breaking $2 M_{W}^{2} / g_{2}^{2}=\left\langle\left|H_{1}\right|^{2}\right\rangle+\left\langle\left|H_{2}\right|^{2}\right\rangle$ Thus at the end of the day we are left essentially with no free parameters. In 31 the consistency of the above boundary conditions with the appropriate radiative electroweak symmetry breaking was explored. Unfortunately, it was found that they are not consistent with the measured value of the top-quark mass, namely the mass obtained in this scheme turns out to be too small. A possible way-out to this situation is to assume that also the moduli fields contribute to SUSY

${ }^{c}$ It is worth remarking that these particular boundary conditions have also interesting fiteness properties. In particular, they preserve one-loop finiteness of $N=1$ finite theories 29. 
breaking, since the soft terms are then modified. Of course, this amounts to a departure of the pure dilaton-dominated scenario. This possibility will be discussed in the context of orbifold models in the next subsection.

Finally, we recall that the phenomenological problem of the pure dilatondominated limit mentioned above is also obtained in a different context, namely from requiring the absence of low-energy charge and color breaking minima deeper than the standard vacuum 32 . In fact, on these grounds, the dilatondominated limit is excluded not only for a $\mu$ term generated through the Kähler potential but for any possible mechanism solving the $\mu$ problem. The results indicate that the whole free parameter space $\left(m_{3 / 2}, B, \mu\right)$ is excluded after imposing the present experimental data on the top mass. Again this rests on the assumption of radiative symmetry breaking with nothing but the MSSM in between the weak scale and the superstring scale.

\section{Dilaton/Moduli SUSY breaking}

In general the moduli fields $T_{i}$ may also contribute to SUSY breaking, i.e. $F^{i} \neq 0$ in ${ }_{1}$ and therefore their effects on soft parameters must also be

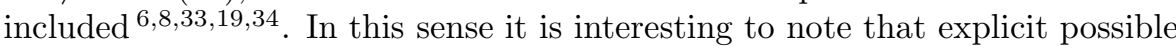
scenarios of SUSY breaking by gaugino condensation in superstrings, when analyzed at the one-loop level, lead to the mandatory inclusion of the moduli in the game (in fact the moduli are the main source of SUSY breaking in these cases) 3 . Since different compactification schemes give rise to different expressions for the moduli-dependent part of the Kähler potential (43), the computation of the bosonic soft parameters will be model dependent. The results are discussed below in the context of some specific superstring models.

\subsection{Superstring models}

To illustrate the main features of mixed dilaton/moduli SUSY breaking, we will concentrate mainly on the case of diagonal moduli and matter metrics. For instance, under this assumption the parametrization (46) is simplified to

$$
\begin{aligned}
F^{S} & =\sqrt{3} C m_{3 / 2} \hat{K}_{\bar{S} S}^{-1 / 2} \sin \theta e^{-i \gamma_{S}}, \\
F^{i} & =\sqrt{3} C m_{3 / 2} \hat{K}_{\bar{i} i}^{-1 / 2} \cos \theta \Theta_{i} e^{-i \gamma_{i}},
\end{aligned}
$$

where $\sum_{i} \Theta_{i}^{2}=1$. Although this is the generic case e.g. in most orbifolds, off-diagonal metrics are present in general in Calabi-Yau compactifications. This may lead to FCNC effects in the low-energy effective $\mathrm{N}=1$ softly broken Lagrangian. The analysis of soft SUSY-breaking parameters in falabiYau compactifications is therefore more involved and can be found in 36 using parametrization (46). A similar analysis for the few orbifolds with off-diagonal 
metrics was carried out in 34 . Some comments about the "off-diagonal" results will be made below. Also in the case of orbifold compactifications with continuous Wilson lines off-diagonal moduli metrics arise, due to the moduli-Wilson line mixing. However, this analysis turns out to be simple 37 and the results are similar to the ones studied below in the diagonal case.

Since the moduli part of the Kähler potential (43) has been computed for $(0,2)$ symmetric Abelian orbifolds, we will concentrate here on these models. They contain generically three $T$-type moduli (the exceptions are the orbifolds $Z_{3}, Z_{4}$ and $Z_{6}^{\prime}$, which have 9,5 and 5 respectively, and are precisely the ones with off-diagonal metrics) and, at most, three $U$-type moduli. We will denote them collectively by $T_{i}$, where e.g. $T_{i}=U_{i-3} ; i=4,5,6$. For this class of models the Kähler potential has the form

$$
K=-\log \left(S+S^{*}\right)-\sum_{i} \log \left(T_{i}+T_{i}^{*}\right)+\sum_{\alpha}\left|C^{\alpha}\right|^{2} \Pi_{i}\left(T_{i}+T_{i}^{*}\right)^{n_{\alpha}^{i}} .
$$

Here $n_{\alpha}^{i}$ are (zero or negative) fractional numbers usually called "modular weights" of the matter fields $C^{\alpha}$. For each given Abelian orbifold, independently of the gauge group or particle content, the possible values of the modular weights are very restricted. For a classification of modular weights for all Abelian orbifolds see 38. The piece proportional to $Z_{\alpha \beta}$ in (43) has been shown to be present in Calabi-Yau compactifications and orbifolds. In particular, in the case of orbifolds, such a term arises when the untwisted sector has at least one complex-structure field $U$ and has been explicitly computed. We will analyze separately this case below, as well as the associated $\mu$ and $B$ parameters, whereas we will concentrate here on the other bosonic soft parameters. Plugging the particular form (59) of the Kähler potential and the parametrization (58) in (22) and (23) we pebtain the following results for the scalar masses and trilinear parameters $34,33,19$ :

$$
\begin{aligned}
m_{\alpha}^{2}= & m_{3 / 2}^{2}\left(1+3 C^{2} \cos ^{2} \theta \overrightarrow{n_{\alpha}} \cdot \overrightarrow{\Theta^{2}}\right)+V_{0} \\
A_{\alpha \beta \gamma}= & -\sqrt{3} C m_{3 / 2}\left(\sin \theta e^{-i \gamma_{S}}+\cos \theta \sum_{i=1}^{6} e^{-i \gamma_{i}} \Theta_{i}[1\right. \\
& \left.\left.+n_{\alpha}^{i}+n_{\beta}^{i}+n_{\gamma}^{i}-\left(T_{i}+T_{i}^{*}\right) \partial_{i} \log Y_{\alpha \beta \gamma}\right]\right) .
\end{aligned}
$$

It is easy to check that the results (49) and (51) are recovered in the limit where $\cos \theta \rightarrow 0$. Notice that neither the scalar (60) nor the gaugino masses (48) have any explicit dependence on $S$ or $T_{i}$ : they only depend on the gravitino mass and the goldstino angles. This is one of the advantages of a parametrization in 
terms of such angles. Although in the case of the $A$-parameter an explicit $T_{i^{-}}$ dependence may appear in the term proportional to $\partial_{i} \log Y_{\alpha \beta \gamma}$, it disappears in several interesting cases 34 . Using the above information, we can now analyze the structure of soft parameters available in Abelian orbifolds.

In the dilaton-dominated case $(\cos \theta=0)$ the soft parameters are universal, as already studied in the previous section. However, in general, they show a lack of universality due to the modular weight dependence (see (60) and (61)). So, even with diagonal matter metrics, FCNC effects may appear. However, we recall that the low-energy running of the scalar masses has to be taken into account. In particular, in the squark case, for gluino masses heavier than (or of the same order as) the scalar masses at the boundary scale, there are large flavour-independent gluino loop contributions which are the dominant source of scalar masses (see (17)). We will show below that this situation is very common in orbifold models. The above effect can therefore help in fulfilling the FCNC constraints.

Another feature of the case under study is that, depending on the goldstino direction, tachyons may appear. For $\cos ^{2} \theta \geq 1 / 3$, the goldstino direction cannot be chosen arbitrarily if one is interested in avoiding tachyons (see (60)). Nevertheless, having a tachyonic sector is not necessarily a problem, it may even be an advantage 34 . In the case of superstring GUTs (or the standard model with extra U(1) interactions), the negative squared mass may just induce gauge symmetry breaking by forcing a VEV for a particular scalar, GUTHiggs field, in the model. The latter possibility provides us with interesting phenomenological consequences: the breaking of SUSY could directly induce further gauge symmetry breaking.

Finally, let us consider three particles $C^{\alpha}, C^{\beta}$ and $C^{\gamma}$, coupled through a Yukawa $Y_{\alpha \beta \gamma}$. They may belong both to the untwisted (U) sector or to a twisted (T) sector, i.e. we consider couplings of the type UUU, UTT, TTT Then, using the above formulae $(60)$ and $(48)$, with negligible $V_{0}$, one finds 34 that in general for any choice of goldstino direction

$$
m_{\alpha}^{2}+m_{\beta}^{2}+m_{\gamma}^{2} \leq\left|M_{a}\right|^{2}=3 m_{3 / 2}^{2} \sin ^{2} \theta .
$$

Remarkably, the same sum rule is fulfilled even in the presence of off-diagonal metrics, as it is the case of the orbifolds $Z_{3}, Z_{4}$ and $Z_{6}^{\prime}$. The three scalar mass eigenvalues will be in general non-degenerate, which in turn may induce FCNC. This can be automatically avoided in the dilaton dominated limit or under special conditions (for instance, when $\hat{W}$ does not depend on the moduli, a no-scale scenario arises and the mass eigenvalues vanish). The same problem is present in Calabi-Yau compactifications, where again the mass eigenvalues are typically non-degenerate. Besides, the sum rule $(62)$ is violated in general 
36. Coming back to the orbifold case, notice that the above sum rule implies that on average scalars are lighter than gauginos. For small $\sin \theta$, some particular scalar mass may become bigger than the gaugino mass, but in that case at least one of the other scalars involved in the sum rule would be forced to have a negative squared mass. This situation is quite dangerous in the context of standard model four-dimensional superstrings, since some observable particles, like Higgses, squarks or sleptons, could be forced to acquire large VEVs (of order the superstring scale). If the above sum rule is applied and squared soft masses are (conservatively) required to be non-negative in order to avoid instabilities of the scalar potential, then the tree level soft masses of observable scalars are constrained to be always smaller than gaugino masses at the boundary scale:

$$
m_{\alpha}<M_{a} .
$$

In turn, this implies that, at low-energy $\left(\sim M_{Z}\right)$, the masses of gluinos, sleptons and squarks (except stops and left sbottom) are ordered as

$$
m_{l}<m_{q} \simeq M_{g},
$$

where gluinos are slightly heavier than scalars. Therefore, in spite of the different set of (non-universal) soft scalar masses, the low-energy phenomenological predictions of the mixed dilaton/moduli SUSY breaking become qualitatively similar to those of the pure dilaton-dominated SUSY breaking. This holds especially for the squark masses, as follows e.g. from (63) and (17). In the case of sleptons, which do not feel gluino loop effects, the boundary values of the soft masses (63) can be relatively more important at low-energy, and larger deviations from the numerical results of (56) can be obtained. Analyses of the low-energy predictions of the dilaton/moduliscenario taking account of the radiative symmetry breaking can be found in 6233.

Before concluding, we recall that exceptions to the above pattern (63), (64) can arise in several situations 34 . For instance, since the total squared Higgs masses receive a positive contribution $\mu^{2}$, the corresponding soft masses may be allowed to be negative: in this case the restrictions from the sum rule would be relaxed. Another example concerns MSSM Yukawa couplings that arise effectively from higher dimension operators: in this case the three-particle sum rule itself may not hold. Finally, a departure from relations (63) and (64) can also arise when both scalar and gaugino masses vanish at tree level. Such a vanishing can happen in the fully moduli-dominated SUSY breaking, e.g. if SUSY breaking is equally shared among $T_{1}, T_{2}, T_{3}$ and one consider untwisted particles: then superstring loop effects become important and tend to make 
scalars heavier than gauginos 1 . In any event, we stress again that potential violations of the pattern in (63) and (64) can occur only when SUSY breaking is mainly moduli dominated (specifically, $\cos ^{2} \theta \geq 2 / 3$ ), since only in this case the gaugino masses can decrease below $m_{3 / 2}$ and possibly become lighter than some scalar mass.

The $B$ parameter and the $\mu$ problem

As already discussed in section 2.1, the two mechanisms to solve the $\mu$ problem in the context of supergravity are naturally present in superstring models. We will concentrate here on the case in which $\mu$ arises from a bilinear term in the Kähler potential (3). The alternative mechanism which generates $\mu$ from the superpotential, as in (16), may also be present in grbifolds, but the results are more model dependent. They can be found in 34 . We recall that, in any orbifold with at least one complex-structure field $U$, the Kähler notential of the untwisted sector possesses the structure $Z\left(T_{i}, T_{i}^{*}\right) C_{1} C_{2}+$ h.c. 14.15 and can therefore generate a $\mu$ term. Specifically, the $Z_{N}$ orbifolds based on $Z_{4}, Z_{6}$, $Z_{8}, Z_{12}^{\prime}$ and the $Z_{N} \times Z_{M}$ orbifolds based on $Z_{2} \times Z_{4}$ and $Z_{2} \times Z_{6}$ do all have a $U$-type field in (say) the third complex plane. In addition, the $Z_{2} \times Z_{2}$ orbifold has $U$ fields in the three complex planes. In all these models the piece of the Kähler potential involving the moduli and the untwisted matter fields $C_{1,2}$ in the third complex plane has the form

$$
\begin{aligned}
K_{3} & =-\log \left[\left(T_{3}+T_{3}^{*}\right)\left(U_{3}+U_{3}^{*}\right)-\left(C_{1}+C_{2}^{*}\right)\left(C_{1}^{*}+C_{2}\right)\right] \\
& \simeq-\log \left(T_{3}+T_{3}^{*}\right)-\log \left(U_{3}+U_{3}^{*}\right)+\frac{\left(C_{1}+C_{2}^{*}\right)\left(C_{1}^{*}+C_{2}\right)}{\left(T_{3}+T_{3}^{*}\right)\left(U_{3}+U_{3}^{*}\right)} .
\end{aligned}
$$

Now, from the expansion shown in (66), one can easily read off the functions $Z, \tilde{K}_{1}, \tilde{K}_{2}$ associated to $C_{1}$ and $C_{2}$ :

$$
Z=\tilde{K}_{1}=\tilde{K}_{2}=\frac{1}{\left(T_{3}+T_{3}^{*}\right)\left(U_{3}+U_{3}^{*}\right)} .
$$

Let us assume that the MSSM can be obtained from a superstring model of the kind mentioned above and let us identify the fields $C_{1}$ and $C_{2}$ with the electroweak Higgs fields $H_{1}$ and $H_{2}$. Plugging back the expressions (67) in (24) and (28) with $\mu=0$, and using the parametrization (58), one can compute $\mu$ and $B$ for this interesting class of models 34 :

$$
\begin{aligned}
\widehat{\mu}= & m_{3 / 2}\left[1+\sqrt{3} C \cos \theta\left(e^{i \gamma_{3}} \Theta_{3}+e^{i \gamma_{6}} \Theta_{6}\right)\right], \\
B \widehat{\mu}= & 2 m_{3 / 2}^{2}\left[1+\sqrt{3} C \cos \theta\left(\cos \gamma_{3} \Theta_{3}+\cos \gamma_{6} \Theta_{6}\right)\right. \\
& \left.+3 C^{2} \cos ^{2} \theta \cos \left(\gamma_{3}-\gamma_{6}\right) \Theta_{3} \Theta_{6}\right]+V_{0} .
\end{aligned}
$$


Notice that, in the limit where $\cos \theta \rightarrow 0$, the results in (57) are recovered. In addition, we recall from (60) that the soft masses are

$$
m_{H_{1}}^{2}=m_{H_{2}}^{2}=m_{3 / 2}^{2}\left[1-3 C^{2} \cos ^{2} \theta\left(\Theta_{3}^{2}+\Theta_{6}^{2}\right)\right]+V_{0} .
$$

In general, the quadratic part of the Higgs potential after SUSY breaking has the form $(\operatorname{see}(21))$

$$
V_{2}=\left(m_{H_{1}}^{2}+|\widehat{\mu}|^{2}\right)\left|\widehat{H}_{1}\right|^{2}+\left(m_{H_{2}}^{2}+|\widehat{\mu}|^{2}\right)\left|\widehat{H}_{2}\right|^{2}+\left(B \widehat{\mu} \widehat{H}_{1} \widehat{H}_{2}+\text { h.c. }\right),
$$

where we recall that $\hat{H}_{1}$ and $\hat{H}_{2}$ are the canonically normalized Higgs fields. In the specific case under consideration, from $(68),(69)$ and $(70)$ we find the remarkable result that the three coefficients in $V_{2}$ are equal, i.e.

$$
m_{H_{1}}^{2}+|\widehat{\mu}|^{2}=m_{H_{2}}^{2}+|\widehat{\mu}|^{2}=B \widehat{\mu} .
$$

so that $V_{2}$ has the simple form

$$
V_{2}=B \widehat{\mu}\left(\widehat{H}_{1}+\widehat{H}_{2}^{*}\right)\left(\widehat{H}_{1}^{*}+\widehat{H}_{2}\right) .
$$

and therefore $\tan \beta=\frac{\left\langle\hat{H}_{2}\right\rangle}{\left\langle\hat{H}_{1}\right\rangle}=-1$. Of course, this corresponds to the boundary condition on the scalar potential at the superstring scale: at lower energies the renormalization group equations should be used. Although the common value of the three coefficients in (72) depends on the Goldstino direction via the parameters $\cos \theta, \Theta_{3}, \Theta_{6}, \ldots$ (see e.g. the expression of $B \widehat{\mu}$ in (69)), we stress that the equality itself and the form of $V_{2}$ hold independently of the Goldstino direction.

Starting from such 'superstringy' boundary conditions for the MSSM parameters gne can explore their consistency with radiative electroweak-symmetry breaking 31 (see also 4 ). One finds that consistency with the measured value of the top-quark mass cannot be achieved in the dilaton-dominated scenario (as already mentioned in section 3.1). The only SUSY-breaking scenario compatible with such constraints requires a suppressed dilaton contribution and important (often dominant) contributions from the $T_{3}, U_{3}$ moduli.

\section{Final Comments and Outlook}

It is worth remarking that most of the above results for soft terms in superstring models refer to certain simple perturbative heterotic compactifications. In addition, it is assumed that the goldstino is a fermionic partner of some combination of the dilaton and/or the moduli fields. Recently some information about the non-perturbative regime in superstring theory has been obtained in terms of the S-dualities 4 of the theory. All superstring theories 
seem to correspond to some points in the parameter space of a unique elevendimensional underlying theory, M-theory 42 . Although the structure of this theory is largely unknown, some preliminary attempts have been made to extract some information of phenomenological interest. A scenario to understand the difference between the GUT scale and the superstring scale has been put forward 43 . Supersymmetry breaking and other phenomenological issues have also been explored within this context in 44 . Studies in progress concerning non-perturbative superstring vacua with $N=1$ SUSY will certainly bring us new surprises.

\section{References}

1. For a review, see: H.P. Nilles, Phys. Rep. 110 (1984) 1, and references therein.

2. For a recent review, see: C. Muñoz, hep-th/9507108, and references therein.

3. For a review, see: C. Muñoz, hep-ph/9601325, and references therein.

4. Y. Kawamura, T. Kobayashi and T. Komatsu, hep-ph/9609468, and references therein.

5. S.K. Soni and H.A. Weldon, Phys. Lett. B126 (1983) 215.

6. A. Brignole, L.E. Ibáñez and C. Muñoz, Nucl. Phys. B422 (1994) 125 [Erratum: B436 (1995) 747].

7. K. Choi, J.E. Kim and H.P. Nilles, Phys. Rev. Lett. 73 (1994) 1758; K. Choi, J.E. Kim and G.T. Park, Nucl. Phys. B442 (1995) 3.

8. S. Ferrara, C. Kounnas and F. Zwirner, Nucl. Phys. B429 (1994) 589 [Erratum: B433 (1995) 255].

9. K. Choi, J.S. Lee and C. Muñoz, hep-ph/9709250.

10. G.F. Giudice and A. Masiero, Phys. Lett. B206 (1988) 480.

11. J.A. Casas and C. Muñoz, Phys. Lett. B306 (1993) 288.

12. J.E. Kim and H.P. Nilles, Phys. Lett. B138 (1984) 150, Phys. Lett. B263 (1991) 79; E.J. Chun, J.E. Kim and H.P. Nilles, Nucl. Phys. B370 (1992) 105.

13. V.S. Kaplunovsky and J. Louis Phys. Lett. B306 (1993) 269.

14. G. Lopes-Cardoso, D. Lüst and T. Mohaupt, Nucl. Phys. B432 (1994) 68.

15. I. Antoniadis, E. Gava, K.S. Narain and T.R. Taylor, Nucl. Phys. B432 (1994) 187.

16. A. Lleyda and C. Muñoz, Phys. Lett. B317 (1993) 82.

17. D. Choudhury, F. Eberlein, A. Köning, J. Louis and S. Pokorski, Phys. Lett. B342 (1995) 180.

18. J. Louis and Y. Nir, Nucl. Phys. B447 (1995) 18.

19. P. Brax and M. Chemtob, Phys.Rev. D51 (1995) 6550. 
20. P. Brax and C.A. Savoy, Nucl. Phys. B447 (1995) 227.

21. For a review, see: A.B. Lahanas and D.V. Nanopoulos, Phys. Rep. 145 (1987) 1, and references therein.

22. J.A. Casas, A. Lleyda and C. Muñoz, Nucl. Phys. B471 (1996) 3, Phys. Lett. B389 (1996) 305.

23. K. Choi, Phys. Rev. Lett. 72 (1994) 1592.

24. B. Acharya, D. Bailin, A. Love, W.A. Sabra and S. Thomas, Phys. Lett. B357 (1995) 387; D. Bailin, G.V. Kraniotis and A. Love, hepth/9705244, hep-th/9707109.

25. A. de la Macorra and G.G. Ross, Nucl. Phys. B404 (1993) 321; V. Halyo and E. Halyo, Phys. Lett. B382 (1996) 89.

26. R. Barbieri, J. Louis and M. Moretti, Phys. Lett. B312 (1993) 451 [Erratum: B316 (1993) 632].

27. J.L. Lopez, D.V. Nanopoulos and A. Zichichi, Phys. Lett. B319 (1993) 451.

28. S. Khalil, A. Masiero and F. Vissani Phys. Lett. B375 (1996) 154.

29. See e.g. L.E. Ibáñez, hep-th/9505098, Proc. of Strings 95, World Scientific (1995), and references therein.

30. J.A. Casas, Phys. Lett. B384 (1996) 103.

31. A. Brignole, L.E. Ibáñez and C. Muñoz, Phys. Lett. B387 (1996) 305.

32. J.A. Casas, A. Lleyda and C. Muñoz, Phys. Lett. B380 (1996) 59.

33. T. Kobayashi, D. Suematsu, K. Yamada and Y. Yamagishi, Phys. Lett. B348 (1995) 402.

34. A. Brignole, L.E. Ibáñez, C. Muñoz and C. Scheich, Z. Phys. C74 (1997) 157.

35. A. Font, L.E. Ibañez, D. Lüst and F. Quevedo, Phys. Lett. B245 (1990) 401; S. Ferrara, N. Magnoli, T.R. Taylor and G. Veneziano, Phys. Lett. B245 (1990) 409; M. Cvetic, A. Font, L.E. Ibañez, D. Lüst and F. Quevedo, Nucl. Phys. B361 (1991) 194; B. de Carlos, J.A. Casas and C. Muñoz, Phys. Lett. B299 (1993) 234, Nucl. Phys. B399 (1993) 623; A. de la Macorra and G.G. Ross, Phys. Lett. B325 (1994) 85.

36. H.B. Kim and C. Muñoz, Z. Phys. C75 (1997) 367.

37. H.B. Kim and C. Muñoz, Mod. Phys. Lett. A12 (1997) 315.

38. L.E. Ibáñez and D. Lüst, Nucl. Phys. B382 (1992) 305.

39. C.-H. Chen, M. Drees and J.F. Gunion, Phys. Rev. D55 (1997) 330; Y. Kawamura, S. Khalil and T. Kobayashi, hep-ph/9703239; A. Love and P. Stadler, hep-ph/9709234

40. Y. Kawamura, T. Kobayashi and M. Watanabe, DPSU-97-5, hepph/9609469.

41. A. Font, L.E. Ibáñez, D. Lüst and F. Quevedo, Phys. Lett. B249 (1990) 
35; A. Sen, Int.J. Mod.Phys. A9 (1994) 3707.

42. See e.g. J.H. Schwarz, hep-th/9607201, P.K. Townsend, hep-th/9612121, and references therein.

43. E. Witten, Nucl. Phys. B471 (1996) 135.

44. T. Banks and M. Dine, hep-th/9605130, hep-th/960819d, hepth/9609046: E. Caceres, V.S. Kaplunovsky and I.M. Mandelberg, hepth/9606036; P. Horava, hep-th/9608019; T. Li, J. Lopez and D.V. Nanopoulos, hep-ph/9702237, hep-ph/9704247; E. Dudas and C. Grojean, hep-th/9704177; I. Antoniadis and M. Quirós, hep-th/9705037, hep-th/9707208; K. Choi, hep-th/9706177; H.P. Nilles, M. Olechowski and M. Yamaguchi, hep-th/970714s; Z. Lalak and S. Thomas, hepth/9707223; V. Kaplunovsky and J. Louis, hep-th/9708049; E. Dudas, hep-th/9709043. 